\title{
In vitro antiplasmodial activity of medicinal plants native to or naturalised in South Africa
}

\author{
Cailean Clarkson $^{\mathrm{a}}$, Vinesh J. Maharaj ${ }^{\mathrm{b}}$, Neil R. Crouch ${ }^{\mathrm{c}, \mathrm{d}}$, Olwen M. Grace ${ }^{\mathrm{c}}$, Pamisha Pillay ${ }^{\mathrm{b}}$, \\ Motlalepula G. Matsabisa ${ }^{\mathrm{e}}$, Niresh Bhagwandin ${ }^{\mathrm{e}}$, Peter J. Smith ${ }^{\mathrm{a}, *}$, Peter I. Folb ${ }^{\mathrm{a}}$ \\ a Pharmacology Division, Department of Medicine, University of Cape Town, K-45 OMB GSH, Observatory 7925, South Africa \\ ${ }^{\mathrm{b}}$ Bio/Chemtek, CSIR, P.O. Box 395, Pretoria 0002, South Africa \\ ${ }^{c}$ Ethnobotany Unit, National Botanical Institute, P.O. Box 52099, Berea Road, Durban 4007, South Africa \\ d Natural Products Research Group, School of Chemistry, University of KwaZulu-Natal, Durban 4041, South Africa \\ ${ }^{\mathrm{e}}$ Medical Research Council, P.O. Box 19070, Tygerberg 7505, South Africa
}

Received 13 November 2003; received in revised form 9 February 2004; accepted 9 February 2004

Available online 20 April 2004

\begin{abstract}
The increasing prevalence and distribution of malaria has been attributed to a number of factors, one of them being the emergence and spread of drug resistant parasites. Efforts are now being directed towards the discovery and development of new chemically diverse antimalarial agents. The present study reports on the in vitro antiplasmodial activity of 134 plant taxa native to or naturalised in South Africa, representing 54 families, which were selected semi-quantitatively using weighted criteria. The plant extracts were tested for in vitro activity against a Plasmodium falciparum strain D10 using the parasite lactate dehydrogenase (pLDH) assay. Of the 134 species assayed, $49 \%$ showed promising antiplasmodial activity $\left(\mathrm{IC}_{50} \leq 10 \mu \mathrm{g} / \mathrm{ml}\right)$, while $17 \%$ were found to be highly active $\left(\mathrm{IC}_{50} \leq 5 \mu \mathrm{g} / \mathrm{ml}\right)$. Several plant species and genera were shown for the first time to possess in vitro antiplasmodial activity. These results support a rational rather than random approach to the selection of antiplasmodial screening candidates, and identify a number of promising taxa for further investigation as plant-based antimalarial agents. (C) 2004 Elsevier Ireland Ltd. All rights reserved.
\end{abstract}

Keywords: Malaria; Antiplasmodial; Plasmodium falciparum; Ethnomedicinal plants; South Africa

\section{Introduction}

Despite intensive efforts to control malaria, the disease continues to be one of the greatest health problems facing Africa. It is estimated that there are at least 300 million clinical cases of malaria per annum, making it one of the top three killers among communicable diseases (WHO, 2003). Although a number of advances have been made towards the understanding of the disease, relatively few antimalarial drugs have been developed in the last 30 years (Ridley, 2002). Since the treatment and control of malaria depends largely on a limited number of chemoprophylactic and chemotherapeutic agents, there is an urgent need to develop novel, affordable antimalarial treatments. This urgency has been further highlighted by the increasing prevalence of drug resistant strains of the malaria parasite Plasmodium falciparum, which have contributed to the escalating disease burden.

\footnotetext{
* Corresponding author. Tel.: +27-21-4066289; fax: +27-21-4481989.

E-mail address: psmith@uctgsh1.uct.ac.za (P.J. Smith).
}

Historically, the majority of antimalarial drugs have been derived from medicinal plants or from structures modelled on plant lead compounds. These include the quinoline-based antimalarials as well as artemisinin and its derivatives. Medicinal plants are commonly used in South African traditional healthcare to treat a range of ailments, including malaria and its associated symptoms (Watt and Breyer-Brandwijk, 1962). Of the 24,300 higher plant taxa recorded in the Flora of Southern Africa (FSA) region, approximately $15 \%$ are used for medicinal purposes (Arnold et al., 2002) and have been documented in various publications and electronic databases. The importance of the region's diverse medicinal plants lies not only in their chemotherapeutic value in traditional healthcare but also in their potential as sources of new chemical entities for drug discovery.

South Africa boasts remarkable biodiversity and rich cultural traditions of plant use. Scientific understanding of medicinal plants is, however, largely unexplored and pharmacological investigation of the South African flora only gained momentum recently (Van Wyk, 2002). 
In light of this and the pressing need for new antimalarial agents, the South African Department of Arts, Culture, Science and Technology (now the Department of Science and Technology) awarded an innovation fund to five South African institutions to evaluate local medicinal plants for antimalarial activity. The aim of this collaborative project was to discover novel, effective plant-based medicines for the treatment of malaria. In this article, we report on the in vitro antiplasmodial activity of 134 species of plants and identify potential sources of new antimalarial drugs.

\section{Materials and methods}

\subsection{Selection and collection of plant material}

A survey of relevant literature (30 books) on medicinal plant use in East and Southern Africa revealed approximately 700 taxa associated with malaria and/or fever. All taxa (623) occurring, indigenous or naturalised within the FSA region, were flagged as potential candidates for a targeted antiplasmodial screen. They were subsequently ranked following the application of weighted criteria, principally ethnobotanical and chemotaxonomic. Similar methodology has previously been applied to the selection of plant molluscicidal candidates from the FSA region (Clark et al., 1997). In the present study, this semi-quantitative approach objectively optimised the selection process and arguably maximised the likelihood of identifying positive antiplasmodial leads. Scores were based on each taxon's association with malaria and/or fever, the documented chemotherapeutic (antiplasmodial) potential of the plant family, use in traditional medicine, occurrence in the regional malaria-endemic area, and popularity in the local ethnomedicinal plant trade. Extra weighting was provided to plants indigenous to the FSA region. From the ranked list, 475 taxa attained total scores of seven or more, of which 134 species were collected throughout South Africa and subsequently investigated. Notably, the top-ranked taxon possessed a total score of 17 out of a possible maximum 20 points. The relevance and suitability of the selection approach and the scoring system will be reported on in due course in a separate publication. Voucher specimens were identified and deposited at the National Herbarium (Pretoria).

\subsection{Preparation of extracts}

Plant samples were separated into different components and dried in an oven at $30-60^{\circ} \mathrm{C}$. The drying time and temperature varied depending on the nature of the plant part. Dried plant material was ground to a coarse powder using a hammer mill and stored at ambient temperature prior to extraction. For each extraction procedure, $100-500 \mathrm{~g}$ of powdered plant material was sequentially extracted, typically with cold dichloromethane (DCM), DCM/methanol $(\mathrm{MeOH})(1: 1), \mathrm{MeOH}$ and purified water. Organic extracts were concentrated by rotary vacuum evaporation below $45^{\circ} \mathrm{C}$ and then further dried in vacuo at ambient temperature for $24 \mathrm{~h}$. The aqueous extracts were concentrated by freeze-drying. All dried extracts were stored at $-20^{\circ} \mathrm{C}$ and the yields of the extracts, in terms of starting plant material, were recorded.

\subsection{In vitro antiplasmodial activity}

A chloroquine-sensitive strain (D10) of Plasmodium falciparum was continuously cultured according to the methods described by Trager and Jensen (1976), and parasite lactate dehydrogenase (pLDH) activity was used to measure parasite viability (Makler et al., 1995). The in vitro assays were performed as previously described by Clarkson et al. (2003). The $\mathrm{IC}_{50}$ values were obtained from the dose-response curves, using non-linear dose-response curve fitting analyses with GraphPad Prism v.3.00 software. Chloroquine diphosphate (Sigma) served as the positive control and was made up in Millipore water and serially diluted in medium to the required concentrations. All crude plant extracts were stored at $-20^{\circ} \mathrm{C}$ prior to testing and stock solutions were made up a day before the experiment and stored at $-20^{\circ} \mathrm{C}$. Crude plant extracts were first dissolved in $\mathrm{MeOH}$ or dimethylsulphoxide (DMSO), depending on their solubility, sonicated for $10 \mathrm{~min}$ and then diluted in Millipore water to prepare a $2 \mathrm{mg} / \mathrm{ml}$ solution. The $2 \mathrm{mg} / \mathrm{ml}$ solution was further diluted in medium to give $200 \mu \mathrm{g} / \mathrm{ml}$ stock solutions. The highest concentration of solvent that the parasites were exposed to was $0.5 \%$, which was shown to have no measurable effect on parasite viability. Extracts were tested in nine serial twofold dilutions (final concentra-

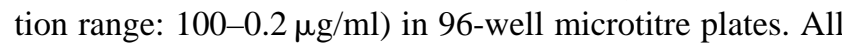
tests were performed in duplicate and no attempt was made to determine $50 \%$ inhibitory concentration $\left(\mathrm{IC}_{50}\right)$ values in excess of $100 \mu \mathrm{g} / \mathrm{ml}$.

\section{Results and discussion}

For the purpose of this study, an $\mathrm{IC}_{50}$ value of $\leq 10 \mu \mathrm{g} / \mathrm{ml}$ was classified as promising activity, and $\leq 5 \mu \mathrm{g} / \mathrm{ml}$ was considered to be highly active. These two concentrations were chosen with the reasoning that inhibition of parasite growth at the low concentrations would indicate selective activity as opposed to higher concentrations where non-specific toxicity is often observed. A total of 134 plant taxa, representing 54 families, were tested for in vitro antiplasmodial activity (Table 1). Sixty-six species showed promising antiplasmodial activity with $\mathrm{IC}_{50}$ values of $\leq 10 \mu \mathrm{g} / \mathrm{ml}$, of which 23 were found to be highly active with $\mathrm{IC}_{50}$ values of $\leq 5 \mu \mathrm{g} / \mathrm{ml}$ (Table 2). Due to the large volumes of data generated in this study, only the highly active extracts are highlighted (Table 2) and further discussed.

Although a substantial amount of phytochemical research has been carried out on Catha edulis (Vahl) Forssk. ex Endl. 
Table 1

In vitro antiplasmodial activity of the plant extracts against Plasmodium falciparum D10

\begin{tabular}{|c|c|c|c|c|c|c|}
\hline Family & Plant species & $\begin{array}{l}\text { Voucher } \\
\text { number }\end{array}$ & Plant part & Solvent & $\%$ Yield & $\begin{array}{l}\mathrm{IC}_{50} \\
(\mu \mathrm{g} / \mathrm{ml})\end{array}$ \\
\hline \multirow[t]{3}{*}{ Acanthaceae } & \multirow[t]{2}{*}{ Asystasia gangetica $\mathrm{T}$. Anderson } & BP00901 & Twigs & $\begin{array}{l}\mathrm{DCM} / \mathrm{MeOH}(1: 1) \\
\text { Water }\end{array}$ & $\begin{array}{l}0.3 \\
7.6\end{array}$ & $\begin{array}{r}16 \\
>100\end{array}$ \\
\hline & & BP00901 & Leaves & $\begin{array}{l}\mathrm{DCM} / \mathrm{MeOH}(1: 1) \\
\text { Water }\end{array}$ & $\begin{array}{l}0.9 \\
1.1\end{array}$ & $\begin{array}{r}7 \\
>100\end{array}$ \\
\hline & Justicia flava (Vahl) Vahl & ВР00960 & Whole plant & $\begin{array}{l}\mathrm{DCM} / \mathrm{MeOH}(1: 1) \\
\text { Water }\end{array}$ & $\begin{array}{l}0.6 \\
3.7\end{array}$ & $\begin{array}{r}31 \\
>100\end{array}$ \\
\hline Amaranthaceae & Achyranthes aspera L. & ВР01260 & Whole plant & $\begin{array}{l}\mathrm{DCM} / \mathrm{MeOH}(1: 1) \\
\text { Water }\end{array}$ & $\begin{array}{l}0.6 \\
0.8\end{array}$ & $\begin{aligned} & 9.9 \\
> & 100\end{aligned}$ \\
\hline Amaryllidaceae & Crinum macowanii Baker & BP00907 & Bulbs & $\begin{array}{l}\mathrm{DCM} / \mathrm{MeOH}(1: 1) \\
\text { Water }\end{array}$ & $\begin{array}{l}0.7 \\
1.1\end{array}$ & $\begin{array}{l}26 \\
25\end{array}$ \\
\hline Anacardiaceae & Lannea discolor (Sond.) Engl. & ВР01389 & Fruit & $\begin{array}{l}\mathrm{DCM} \\
\mathrm{MeOH} / \mathrm{DCM} \\
\text { Water }\end{array}$ & $\begin{array}{l}0.4 \\
0.6 \\
1.1\end{array}$ & $\begin{array}{r}25 \\
>100 \\
>100\end{array}$ \\
\hline \multirow[t]{5}{*}{ Annonaceae } & \multirow[t]{2}{*}{$\begin{array}{l}\text { Annona senegalensis Pers. } \\
\text { subsp. senegalensis }\end{array}$} & \multirow[t]{2}{*}{ EN00334 } & Leaves & $\mathrm{DCM}$ & 0.7 & 35 \\
\hline & & & & DCM/MeOH (1:1) & 0.9 & 45 \\
\hline & Artabotrys brachypetalus Benth. & ВР00210 & Leaves/twigs & $\begin{array}{l}\mathrm{DCM} / \mathrm{MeOH}(1: 1) \\
\text { Water }\end{array}$ & $\begin{array}{l}0.9 \\
1.3\end{array}$ & $\begin{array}{l}>100 \\
>100\end{array}$ \\
\hline & Artabotrys monteiroae Oliv. & BP01273 & Twigs & $\begin{array}{l}\mathrm{DCM} / \mathrm{MeOH}(1: 1) \\
\text { Water }\end{array}$ & $\begin{array}{l}1.2 \\
4.8\end{array}$ & $\begin{aligned} & 8.7 \\
> & 100\end{aligned}$ \\
\hline & & BP01273 & Leaves & $\begin{array}{l}\mathrm{DCM} / \mathrm{MeOH}(1: 1) \\
\text { Water }\end{array}$ & $\begin{array}{l}1.3 \\
0.7\end{array}$ & $\begin{array}{l}22 \\
23\end{array}$ \\
\hline Apiaceae & $\begin{array}{l}\text { Alepidea amatymbica } \text { Eckl. \& } \\
\text { Zeyh. }\end{array}$ & EN00994 & Whole plant & $\mathrm{DCM} / \mathrm{MeOH}(1: 1)$ & 0.7 & 12.5 \\
\hline & & & & Water & 1.7 & $>100$ \\
\hline & Berula erecta (Huds.) Coville & ВР00957 & Whole plant & $\mathrm{DCM} / \mathrm{MeOH}(1: 1)$ & 0.3 & 6.6 \\
\hline & & & & Water & 2.7 & $>100$ \\
\hline & Centella asiatica (L.) Urb. & ВР00955 & Leaves & $\mathrm{DCM} / \mathrm{MeOH}(1: 1)$ & 0.4 & 8.3 \\
\hline Apocynaceae & Carissa edulis Vahl & EN00097 & Stems & $\begin{array}{l}\text { DCM } \\
\text { DCM/MeOH (1:1) }\end{array}$ & $\begin{array}{l}0.6 \\
0.9\end{array}$ & $\begin{array}{l}33 \\
60\end{array}$ \\
\hline & Carissa edulis Vahl & EN00097 & Stems & $\begin{array}{l}\mathrm{MeOH} \\
\text { Water }\end{array}$ & $\begin{array}{l}0.5 \\
1.5\end{array}$ & $\begin{array}{r}100 \\
>100\end{array}$ \\
\hline & & EN00097 & Seeds & DCM & 0.7 & $>100$ \\
\hline & & & & $\mathrm{DCM} / \mathrm{MeOH}(1: 1)$ & 0.9 & $>100$ \\
\hline & & & & $\mathrm{MeOH}$ & 0.9 & $>100$ \\
\hline & & & & Water & 0.6 & $>100$ \\
\hline & & EN00097 & Leaves & DCM & 1.1 & 100 \\
\hline & & & & DCM/MeOH $(1: 1)$ & 0.8 & $>100$ \\
\hline & & & & $\mathrm{MeOH}$ & 0.9 & $>100$ \\
\hline & & & & Water & 1.3 & $>100$ \\
\hline & $\begin{array}{l}\text { Diplorhynchus condylocarpon } \\
\text { (Müll. Arg.) Pichon }\end{array}$ & EN00300 & Roots & DCM & 0.6 & $>100$ \\
\hline & & & & $\mathrm{DCM} / \mathrm{MeOH}(1: 1)$ & 0.9 & 24 \\
\hline & & & & Water & 0.8 & $>100$ \\
\hline & Rauvolfia caffra Sond. & EN00232 & Fruit & DCM & 0.8 & 26.5 \\
\hline & & & & DCM/MeOH (1:1) & 0.3 & 55 \\
\hline & & & & Water & 1.1 & $>100$ \\
\hline & & EN00232 & Roots & $\mathrm{DCM}$ & 1.3 & 88 \\
\hline & & & & DCM/MeOH $(1: 1)$ & 0.9 & 67 \\
\hline & & & & Water & 1.2 & 90 \\
\hline & $\begin{array}{l}\text { Gomphocarpus fruticosus (L.) } \\
\text { Aiton. f. }\end{array}$ & ВP01151 & Fruit & $\mathrm{DCM} / \mathrm{MeOH}(1: 1)$ & 0.6 & 26 \\
\hline Araliaceae & Cussonia spicata Thunb. & ВР00031 & Leaves & $\mathrm{DCM}$ & 1.6 & 45 \\
\hline & & & & $\mathrm{DCM} / \mathrm{MeOH}(1: 1)$ & 0.9 & 13 \\
\hline & & & & $\mathrm{MeOH}$ & 1.0 & 27.5 \\
\hline & & & & Water & 1.8 & 90 \\
\hline & & EN00867 & Fruit & $\mathrm{DCM} / \mathrm{MeOH}(1: 1)$ & 0.4 & 14 \\
\hline & & & & Water & 1.2 & $>100$ \\
\hline
\end{tabular}


Table 1 (Continued)

\begin{tabular}{|c|c|c|c|c|c|c|}
\hline Family & Plant species & $\begin{array}{l}\text { Voucher } \\
\text { number }\end{array}$ & Plant part & Solvent & $\%$ Yield & $\begin{array}{l}\mathrm{IC}_{50} \\
(\mu \mathrm{g} / \mathrm{ml})\end{array}$ \\
\hline & $\begin{array}{l}\text { Schefflera umbellifera (Sond.) } \\
\text { Baill. }\end{array}$ & EN00060 & Leaves & DCM & 0.6 & 3.7 \\
\hline & & & & DCM/MeOH (1:1) & 0.6 & 19.5 \\
\hline & & & & $\mathrm{MeOH}$ & 0.9 & 49.5 \\
\hline & $\begin{array}{l}\text { Schefflera umbellifera (Sond.) } \\
\text { Baill. }\end{array}$ & EN00060 & Leaves & Water & 1.2 & $>100$ \\
\hline & & EN00060 & Roots & DCM & 1.1 & 7.5 \\
\hline & & & & DCM/MeOH (1:1) & 0.9 & 5.8 \\
\hline & & & & $\mathrm{MeOH}$ & 0.7 & $>100$ \\
\hline & & & & Water & 1.0 & $>100$ \\
\hline & & EN00060 & Stems & $\mathrm{DCM}$ & 0.8 & 42 \\
\hline & & & & DCM/MeOH (1:1) & 0.9 & 15 \\
\hline & & & & $\mathrm{MeOH}$ & 0.6 & 35 \\
\hline & & & & Water & 0.9 & 100 \\
\hline & & & & Water & 1.2 & $>100$ \\
\hline & & BP01151 & Leaves/twigs & DCM/MeOH (1:1) & 1.2 & 25 \\
\hline & & & & Water & 0.9 & $>100$ \\
\hline & Xysmalobium undulatum (L.) & ВР01179 & Whole plant & DCM/MeOH (1:1) & 1.4 & 6 \\
\hline & Aiton. f. & & & & & \\
\hline & & & & Water & 1.8 & $>100$ \\
\hline Asparagaceae & Asparagus virgatus Baker & ВР01029 & Whole plant & $\mathrm{DCM} / \mathrm{MeOH}(1: 1)$ & 0.7 & 8 \\
\hline & & & & Water & 0.2 & $>100$ \\
\hline Asphodelaceae & Aloe ferox Mill. & ВР00469 & Whole plant & $\mathrm{DCM} / \mathrm{MeOH}(1: 1)$ & 0.7 & 8 \\
\hline & & & & Water & 1.1 & $>100$ \\
\hline & & EN00588 & Fruit & $\mathrm{DCM}$ & 0.4 & 20 \\
\hline & & & & DCM/MeOH (1:1) & 0.8 & 14 \\
\hline & & & & Water & 0.6 & 18 \\
\hline & & EN00588 & Stems & $\mathrm{DCM}$ & 0.8 & 30 \\
\hline & & & & DCM/MeOH (1:1) & 0.8 & 15.5 \\
\hline & & & & Water & 0.9 & $>100$ \\
\hline & & EN00588 & Roots & DCM & 0.7 & 13 \\
\hline & & & & $\mathrm{DCM} / \mathrm{MeOH}(1: 1)$ & 0.8 & 8.5 \\
\hline & & & & Water & 0.5 & $>100$ \\
\hline & Aloe ferox Mill. & EN00588 & Leaves & DCM & 0.8 & 21 \\
\hline & & & & DCM/MeOH (1:1) & 0.4 & $>100$ \\
\hline & & & & Water & 0.7 & $>100$ \\
\hline & Aloe maculata All. & BP01314 & Whole plant & DCM/MeOH (1:1) & 0.6 & 12.4 \\
\hline & & & & Water & 3.0 & $>100$ \\
\hline & Aloe marlothii A. Berger & ВР00049 & Leaves & DCM & 0.8 & 74 \\
\hline & & & & DCM/MeOH (1:1) & 0.6 & 90 \\
\hline & & & & $\mathrm{MeOH}$ & 0.6 & $>100$ \\
\hline & & & & Water & 0.8 & 71 \\
\hline & & ВР00049 & Whole plant & DCM & 0.4 & 3.5 \\
\hline & & & & DCM/MeOH (1:1) & 0.7 & 18 \\
\hline & & & & Water & 1.0 & $>100$ \\
\hline & Ageratum conyzoides $\mathrm{L}$. & BP01233 & Whole plant & $\mathrm{DCM} / \mathrm{MeOH}(1: 1)$ & 1.4 & 27 \\
\hline & & & & Water & 0.9 & $>100$ \\
\hline Asteraceae & Artemisia afra Jacq. ex Willd. & EN00148 & Leaves & $\mathrm{DCM}$ & 0.7 & 5 \\
\hline & & & & DCM/MeOH (1:1) & 0.9 & 7.3 \\
\hline & & & & $\mathrm{MeOH}$ & 0.9 & 8 \\
\hline & & & & Water & 0.6 & $>100$ \\
\hline & Bidens pilosa $\mathrm{L}$. & EN00001 & Leaves & DCM & 0.4 & 8.5 \\
\hline & & & & $\mathrm{DCM} / \mathrm{MeOH}(1: 1)$ & 0.8 & 11 \\
\hline & & & & $\mathrm{MeOH}$ & 0.6 & 5 \\
\hline & & & & Water & 1.6 & 70 \\
\hline & Conyza albida Spreng. & BP01221 & Whole plant & DCM/MeOH (1:1) & 0.7 & 2 \\
\hline & & & & Water & 1.1 & $>100$ \\
\hline & Conyza podocephala DC. & BP00378 & Whole plant & DCM/MeOH (1:1) & 0.6 & 6.8 \\
\hline & & & & Water & 1.3 & $>100$ \\
\hline & Conyza scabrida DC. & BP00443 & Flowers & DCM/MeOH (1:1) & 0.3 & 7.8 \\
\hline & & & & Water & 0.4 & $>100$ \\
\hline
\end{tabular}


Table 1 (Continued)

\begin{tabular}{|c|c|c|c|c|c|c|}
\hline Family & Plant species & $\begin{array}{l}\text { Voucher } \\
\text { number }\end{array}$ & Plant part & Solvent & $\%$ Yield & $\begin{array}{l}\mathrm{IC}_{50} \\
(\mu \mathrm{g} / \mathrm{ml})\end{array}$ \\
\hline & \multirow[t]{4}{*}{ Conyza scabrida DC. } & \multirow[t]{2}{*}{ BP00443 } & \multirow[t]{2}{*}{ Leaves } & $\mathrm{DCM} / \mathrm{MeOH}(1: 1)$ & 0.6 & 11.5 \\
\hline & & & & Water & 2.2 & $>100$ \\
\hline & & \multirow[t]{2}{*}{ ВP00443 } & \multirow[t]{2}{*}{ Twigs } & $\mathrm{DCM} / \mathrm{MeOH}(1: 1)$ & 0.7 & 11 \\
\hline & & & & Water & 1.1 & $>100$ \\
\hline & \multirow[t]{2}{*}{$\begin{array}{l}\text { Helichrysum nudifolium (L.) } \\
\text { Less. }\end{array}$} & \multirow[t]{2}{*}{ EN01023 } & \multirow[t]{2}{*}{ Whole plant } & $\mathrm{DCM} / \mathrm{MeOH}(1: 1)$ & 0.5 & 6.8 \\
\hline & & & & Water & 2.4 & $>100$ \\
\hline & \multirow[t]{2}{*}{ Osteospermum imbricatum $\mathrm{L}$. } & \multirow[t]{2}{*}{ EN00874 } & \multirow[t]{2}{*}{ Stems } & $\mathrm{DCM} / \mathrm{MeOH}(1: 1)$ & 1.2 & 7.3 \\
\hline & & & & Water & 0.7 & $>100$ \\
\hline & \multirow[t]{9}{*}{ Pentzia globosa Less. } & \multirow[t]{3}{*}{ EN00506 } & \multirow[t]{3}{*}{ Leaves } & DCM & 0.7 & 12.5 \\
\hline & & & & $\mathrm{DCM} / \mathrm{MeOH}(1: 1)$ & 0.6 & 19.5 \\
\hline & & & & Water & 1.2 & 53 \\
\hline & & \multirow[t]{3}{*}{ EN00506 } & \multirow[t]{3}{*}{ Stems } & DCM & 0.9 & 9.5 \\
\hline & & & & DCM/MeOH (1:1) & 0.9 & 15.5 \\
\hline & & & & Water & 0.6 & $>100$ \\
\hline & & EN00393 & Roots & $\mathrm{DCM}$ & 1.1 & 8 \\
\hline & & & & $\mathrm{DCM} / \mathrm{MeOH}(1: 1)$ & 0.8 & 14 \\
\hline & & & & Water & 0.8 & $>100$ \\
\hline & $\begin{array}{l}\text { Psiadia punctulata (DC.) Oliv. } \\
\text { \& Hiern ex Vatke }\end{array}$ & CS00005 & Twigs & DCM & 0.3 & 9 \\
\hline & & & & Water & 0.9 & $>100$ \\
\hline & & $\mathrm{CN} 00044$ & Leaves & DCM & 1.2 & 14 \\
\hline & & & & DCM/MeOH $(1: 1)$ & 1.4 & 22.5 \\
\hline & & & & Water & 0.6 & $>100$ \\
\hline & & BP00278 & Whole plant & DCM/MeOH (1:1) & 1.2 & 18 \\
\hline & & & & Water & 0.9 & $>100$ \\
\hline & Senecio oxyriifolius DC. & BP01037 & Whole plant & DCM/MeOH $(1: 1)$ & 0.7 & 13 \\
\hline & & & & Water & 2.6 & $>100$ \\
\hline & Spilanthes mauritiana (Pers.) & EN00075 & Stems & $\mathrm{DCM}$ & 0.8 & 38 \\
\hline & DC. & & & & & \\
\hline & & & & $\mathrm{DCM} / \mathrm{MeOH}(1: 1)$ & 0.9 & 5.3 \\
\hline & Spilanthes mauritiana (Pers.) & EN00075 & Stems & $\mathrm{MeOH}$ & 0.8 & 64 \\
\hline & DC. & & & & & \\
\hline & & & & Water & 2.6 & $>100$ \\
\hline & Tarchonanthus camphoratus L. & BP00263 & Whole plant & DCM/MeOH (1:1) & 0.6 & 6 \\
\hline & & & & Water & 0.9 & $>100$ \\
\hline & & EN00389 & Leaves & DCM & 0.9 & 7.5 \\
\hline & & & & DCM/MeOH (1:1) & 1.2 & 13 \\
\hline & & & & Water & 1.8 & $>100$ \\
\hline & & EN00389 & Roots & DCM & 1.1 & 60 \\
\hline & & & & DCM/MeOH $(1: 1)$ & 0.8 & 24 \\
\hline & & & & Water & 0.7 & $>100$ \\
\hline & Tridax procumbens $\mathrm{L}$. & BP00892 & Whole plant & DCM/MeOH (1:1) & 0.4 & 17 \\
\hline & & & & Water & 1.9 & $>100$ \\
\hline & Vernonia colorata (Willd.) & BP01279 & Twigs & DCM/MeOH $(1: 1)$ & 0.4 & 14.1 \\
\hline & Drake subsp. colorata & & & & & \\
\hline & & & & Water & 0.6 & $>100$ \\
\hline & & ВР01279 & Leaves & $\mathrm{DCM} / \mathrm{MeOH}(1: 1)$ & 1.5 & 4.7 \\
\hline & & & & Water & 1.0 & 87.7 \\
\hline & Vernonia fastigiata Oliv. \& Hiern & EN00118 & Leaves & $\mathrm{DCM}$ & 1.0 & 10 \\
\hline & & & & $\mathrm{DCM} / \mathrm{MeOH}(1: 1)$ & 0.8 & 15 \\
\hline & & & & $\mathrm{MeOH}$ & 0.6 & 30 \\
\hline & & & & Water & 0.9 & $>100$ \\
\hline & $\begin{array}{l}\text { Vernonia hirsuta (DC.) Sch. } \\
\text { Bip. ex Walp. }\end{array}$ & EN01012 & Whole plant & DCM/MeOH (1:1) & 0.7 & 14 \\
\hline & & & & Water & 1.2 & $>100$ \\
\hline & Vernonia myriantha Hook. f. & EN00044 & Roots & DCM & 0.9 & 61 \\
\hline & & & & $\mathrm{DCM} / \mathrm{MeOH}(1: 1)$ & 0.8 & 37.5 \\
\hline & & & & $\mathrm{MeOH}$ & 0.8 & 28 \\
\hline & & & & Water & 2.3 & 70 \\
\hline & & EN00044 & Leaves & DCM & 0.6 & 3 \\
\hline & & & & DCM/MeOH (1:1) & 0.8 & 13.5 \\
\hline
\end{tabular}


Table 1 (Continued)

\begin{tabular}{|c|c|c|c|c|c|c|}
\hline Family & Plant species & $\begin{array}{l}\text { Voucher } \\
\text { number }\end{array}$ & Plant part & Solvent & $\%$ Yield & $\begin{array}{l}\mathrm{IC}_{50} \\
(\mu \mathrm{g} / \mathrm{ml})\end{array}$ \\
\hline & Vernonia myriantha Hook. f. & EN00044 & Leaves & Water & 2.1 & $>100$ \\
\hline & $\begin{array}{l}\text { Vernonia natalensis Sch. Bip. ex } \\
\text { Walp. }\end{array}$ & EN00331 & Whole plant & DCM & 1.5 & 19.5 \\
\hline & & & & DCM/MeOH (1:1) & 0.7 & 24 \\
\hline & & & & Water & 1.1 & $>100$ \\
\hline & Vernonia oligocephala (DC.) & EN00314 & Roots & $\mathrm{DCM}$ & 0.7 & $>100$ \\
\hline & Sch. Bip. ex Walp. & & & & & \\
\hline & & & & DCM/MeOH $(1: 1)$ & 0.7 & 20 \\
\hline & & & & Water & 2.1 & $>100$ \\
\hline & & EN00314 & Leaves & $\mathrm{DCM}$ & 1.2 & 3.5 \\
\hline & & & & DCM/MeOH $(1: 1)$ & 0.8 & 5.5 \\
\hline & & & & Water & 2.2 & $>100$ \\
\hline \multirow[t]{8}{*}{ Bignoniaceae } & Kigelia africana (Lam.) Benth. & EN00217 & Leaves & DCM & 0.7 & 51 \\
\hline & & & & DCM/MeOH $(1: 1)$ & 0.8 & 87 \\
\hline & & & & Water & 1.7 & $>100$ \\
\hline & Tecomaria capensis (Thunb.) & BP01302 & Leaves & $\mathrm{DCM} / \mathrm{MeOH}(1: 1)$ & 0.1 & 11.6 \\
\hline & Lindl. & & & & & \\
\hline & & & & Water & 3.1 & 10.9 \\
\hline & & BP01302 & Twigs & DCM/MeOH $(1: 1)$ & 0.1 & 81.9 \\
\hline & & & & Water & 2.0 & 10.2 \\
\hline \multirow[t]{9}{*}{ Capparaceae } & Capparis tomentosa Lam. & EN00222 & Leaves & $\mathrm{DCM}$ & 0.5 & 65 \\
\hline & & & & DCM/MeOH (1:1) & 0.9 & $>100$ \\
\hline & & & & Water & 0.7 & $>100$ \\
\hline & & EN00222 & Stems & DCM & 0.4 & 41.5 \\
\hline & & & & DCM/MeOH (1:1) & 0.7 & $>100$ \\
\hline & & & & Water & 0.9 & $>100$ \\
\hline & & EN00222 & Roots & DCM & 0.4 & 38 \\
\hline & & & & DCM/MeOH (1:1) & 0.8 & $>100$ \\
\hline & & & & Water & 1.2 & $>100$ \\
\hline \multirow[t]{31}{*}{ Celastraceae } & $\begin{array}{l}\text { Catha edulis (Vahl) Forssk. ex } \\
\text { Endl. }\end{array}$ & EN00159 & Seeds & DCM & 0.3 & 46 \\
\hline & $\begin{array}{l}\text { Catha edulis (Vahl) Forssk. ex } \\
\text { Endl. }\end{array}$ & EN00159 & Seeds & DCM/MeOH (1:1) & 0.4 & 10 \\
\hline & & & & $\mathrm{MeOH}$ & 0.4 & 78.5 \\
\hline & & & & Water & 0.3 & $>100$ \\
\hline & & EN00159 & Roots & DCM & 0.6 & 0.63 \\
\hline & & & & DCM/MeOH (1:1) & 0.8 & 4.8 \\
\hline & & & & $\mathrm{MeOH}$ & 0.6 & 23.5 \\
\hline & & & & Water & 1.2 & $>100$ \\
\hline & & EN00159 & Leaves & DCM & 0.9 & 0.77 \\
\hline & & & & DCM/MeOH (1:1) & 1.1 & 6.9 \\
\hline & & & & $\mathrm{MeOH}$ & 1.0 & 7.7 \\
\hline & & & & Water & 1.4 & $>100$ \\
\hline & Maytenus senegalensis (Lam.) & EN00218 & Roots & DCM & 2.3 & 15.5 \\
\hline & Exell. & & & & & \\
\hline & & & & Water & 1.2 & $>100$ \\
\hline & & EN00218 & Stems & DCM & 2.7 & 42 \\
\hline & & & & DCM/MeOH (1:1) & 1.3 & 48.3 \\
\hline & & & & Water & 0.4 & $>100$ \\
\hline & Maytenus undata (Thunb.) & EN00029 & Leaves & $\mathrm{DCM}$ & 0.5 & $>100$ \\
\hline & Blakelock & & & & & \\
\hline & & & & DCM/MeOH (1:1) & 0.7 & 21 \\
\hline & & & & $\mathrm{MeOH}$ & 0.7 & 60 \\
\hline & & & & Water & 1.1 & $>100$ \\
\hline & & EN00029 & Stems & DCM & 0.5 & 85 \\
\hline & & & & DCM/MeOH (1:1) & 0.6 & 24 \\
\hline & & & & $\mathrm{MeOH}$ & 0.5 & 38 \\
\hline & & & & Water & 2.1 & $>100$ \\
\hline & & EN00029 & Roots & DCM & 0.6 & 23 \\
\hline & & & & DCM/MeOH (1:1) & 0.8 & 36 \\
\hline & & & & $\mathrm{MeOH}$ & 0.6 & 40 \\
\hline & & & & Water & 2.1 & $>100$ \\
\hline
\end{tabular}


Table 1 (Continued)

\begin{tabular}{|c|c|c|c|c|c|c|}
\hline Family & Plant species & $\begin{array}{l}\text { Voucher } \\
\text { number }\end{array}$ & Plant part & Solvent & $\%$ Yield & $\begin{array}{l}\mathrm{IC}_{50} \\
(\mu \mathrm{g} / \mathrm{ml})\end{array}$ \\
\hline \multirow[t]{8}{*}{ Chrysobalanaceae } & $\begin{array}{l}\text { Parinari curatellifolia Planch. } \\
\text { ex Benth. }\end{array}$ & EN00065 & Leaves/flowers & DCM & 0.8 & 17 \\
\hline & & & & DCM/MeOH (1:1) & 0.7 & 40 \\
\hline & & & & $\mathrm{MeOH}$ & 0.7 & 46.5 \\
\hline & & & & Water & 1.6 & 81 \\
\hline & & EN00065 & Roots & DCM & 0.6 & 5.3 \\
\hline & & & & $\mathrm{DCM} / \mathrm{MeOH}(1: 1)$ & 1.2 & 22.5 \\
\hline & & & & $\mathrm{MeOH}$ & 0.9 & 30.5 \\
\hline & & & & Water & 1.9 & 63.5 \\
\hline \multirow[t]{2}{*}{ Clusiaceae } & Hypericum aethiopicum Thunb. & BP00365 & Leaves/flowers & DCM/MeOH (1:1) & 0.7 & 1.4 \\
\hline & & & & Water & 1.4 & 90 \\
\hline \multirow[t]{2}{*}{ Colchicaceae } & Gloriosa superba $\mathrm{L}$. & BP01040 & Whole plant & DCM/MeOH (1:1) & 0.8 & 17 \\
\hline & & & & Water & 3.7 & $>100$ \\
\hline \multirow[t]{2}{*}{ Combretaceae } & Combretum zeyheri Sond. & ВР01239 & Twigs & $\mathrm{DCM} / \mathrm{MeOH}(1: 1)$ & 0.4 & 15 \\
\hline & & & & Water & 1.2 & $>100$ \\
\hline \multirow[t]{8}{*}{ Cucurbitaceae } & Momordica balsamina $\mathrm{L}$. & FP00062 & Whole plant & DCM/MeOH $(1: 1)$ & 0.9 & 18 \\
\hline & & & & Water & 2.1 & $>100$ \\
\hline & & MM00075 & Stem & DCM/MeOH (1:1) & 0.7 & 5.3 \\
\hline & & & & Water & 0.8 & $>100$ \\
\hline & & MM00075 & Leaves & DCM/MeOH (1:1) & 0.7 & 6 \\
\hline & & & & Water & 2.3 & $>100$ \\
\hline & $\begin{array}{l}\text { Zehneria scabra (L.f.) Sond. } \\
\text { subsp. scabra }\end{array}$ & BP01065 & Whole plant & $\mathrm{DCM} / \mathrm{MeOH}(1: 1)$ & 0.9 & 5.6 \\
\hline & & & & Water & 3.8 & $>100$ \\
\hline \multirow[t]{8}{*}{ Ebenaceae } & Euclea natalensis A.DC. & EN00760 & Stems & DCM/MeOH (1:1) & 1.4 & 5.3 \\
\hline & & & & Water & 2.7 & $>100$ \\
\hline & & EN00760 & Roots & $\mathrm{DCM} / \mathrm{MeOH}(1: 1)$ & 0.9 & 5.1 \\
\hline & & & & Water & 1.3 & $>100$ \\
\hline & Euclea undulata Thunb. & BP01105 & Leaves & DCM/MeOH (1:1) & 1.7 & 11 \\
\hline & & & & Water & 1.1 & $>100$ \\
\hline & Euclea undulata Thunb. & BP01105 & Twigs & DCM/MeOH (1:1) & 1.2 & 4.6 \\
\hline & & & & Water & 0.4 & $>100$ \\
\hline \multirow[t]{24}{*}{ Euphorbiaceae } & Bridelia micrantha (Hochst.) & BP01316 & Twigs & DCM/MeOH (1:1) & 0.5 & 59.3 \\
\hline & Baill. & & & & & \\
\hline & & & & Water & 1.1 & 6.9 \\
\hline & Clutia hirsuta E. Mey. ex Sond. & BP01193 & Whole plant & $\mathrm{DCM} / \mathrm{MeOH}(1: 1)$ & 1.0 & 15 \\
\hline & & & & Water & 2.3 & 50 \\
\hline & $\begin{array}{l}\text { Croton gratissimus Burch. var. } \\
\text { subgratissimus (Prain) Burtt } \\
\text { Davy }\end{array}$ & ВР00929 & Leaves & $\mathrm{DCM}$ & 0.7 & 3.5 \\
\hline & & & & DCM/MeOH (1:1) & 1.2 & 11.5 \\
\hline & & & & $\mathrm{MeOH}$ & 1.1 & 29 \\
\hline & & & & Water & 2.3 & 95 \\
\hline & Croton menyhartii Pax & BP01213 & Leaves & DCM/MeOH (1:1) & 2.0 & 1.7 \\
\hline & & & & Water & 2.0 & $>100$ \\
\hline & & ВР01213 & Twigs & $\mathrm{DCM} / \mathrm{MeOH}(1: 1)$ & 0.8 & 15 \\
\hline & & & & Water & 0.7 & $>100$ \\
\hline & Euphorbia heterophylla $\mathrm{L}$. & BP01249 & Whole plant & DCM/MeOH (1:1) & 1.3 & 40 \\
\hline & & & & Water & 4.8 & $>100$ \\
\hline & Euphorbia tirucalli $\mathrm{L}$. & RP00008 & Leaves & $\mathrm{DCM}$ & 0.8 & 12 \\
\hline & & & & DCM/MeOH (1:1) & 1.2 & 23.5 \\
\hline & & & & $\mathrm{MeOH}$ & 0.7 & $>100$ \\
\hline & & & & Water & 2.3 & 83 \\
\hline & $\begin{array}{l}\text { Flueggea virosa (Roxb. ex } \\
\text { Willd.) }\end{array}$ & ВP00207 & Leaves/twigs & $\mathrm{DCM} / \mathrm{MeOH}(1: 1)$ & 0.3 & 19 \\
\hline & Voigt subsp. virosa & & & Water & 1.4 & 11.4 \\
\hline & $\begin{array}{l}\text { Ricinus communis L. var. } \\
\text { communis }\end{array}$ & EN00768 & Leaves & DCM/MeOH (1:1) & 1.0 & 27.5 \\
\hline & & & & Water & 0.6 & $>100$ \\
\hline & & EN00768 & Stems & DCM/MeOH (1:1) & 0.9 & 8 \\
\hline
\end{tabular}


Table 1 (Continued)

\begin{tabular}{|c|c|c|c|c|c|c|}
\hline Family & Plant species & $\begin{array}{l}\text { Voucher } \\
\text { number }\end{array}$ & Plant part & Solvent & $\%$ Yield & $\begin{array}{l}\mathrm{IC}_{50} \\
(\mu \mathrm{g} / \mathrm{ml})\end{array}$ \\
\hline & & & & Water & 1.3 & $>100$ \\
\hline & & EN00768 & Fruit & DCM/MeOH (1:1) & 0.5 & 90 \\
\hline & & & & Water & 0.6 & $>100$ \\
\hline \multirow[t]{45}{*}{ Fabaceae } & $\begin{array}{l}\text { Acacia nilotica (L.) Willd. ex } \\
\text { Delile }\end{array}$ & ВР01209 & Twigs & $\mathrm{DCM} / \mathrm{MeOH}(1: 1)$ & 0.4 & 13 \\
\hline & $\begin{array}{l}\text { Acacia nilotica (L.) Willd. ex } \\
\text { Delile }\end{array}$ & ВР01209 & Twigs & Water & 6.8 & 32 \\
\hline & Acacia tortilis (Forssk) Hayne & BP01203 & Whole plant & DCM/MeOH (1:1) & 0.7 & 4.8 \\
\hline & & & & Water & 0.1 & $>100$ \\
\hline & Crotalaria burkeana Benth. & EN00126 & Leaves & $\mathrm{DCM}$ & 0.7 & 30 \\
\hline & & & & $\mathrm{DCM} / \mathrm{MeOH}(1: 1)$ & 1.3 & 50 \\
\hline & & & & $\mathrm{MeOH}$ & 1.5 & $>100$ \\
\hline & & & & Water & 0.8 & $>100$ \\
\hline & & EN00126 & Roots & DCM & 0.5 & 9.5 \\
\hline & & & & $\mathrm{DCM} / \mathrm{MeOH}(1: 1)$ & 0.9 & 13 \\
\hline & & & & $\mathrm{MeOH}$ & 1.3 & $>100$ \\
\hline & & & & Water & 1.1 & $>100$ \\
\hline & $\begin{array}{l}\text { Elephantorrhiza elephantina } \\
\text { (Burch.) Skeels }\end{array}$ & ВР01010 & Roots & DCM/MeOH (1:1) & 0.4 & 28 \\
\hline & & & & Water & 1.5 & $>100$ \\
\hline & & BP01010 & Leaves & $\mathrm{DCM} / \mathrm{MeOH}(1: 1)$ & 0.1 & 26 \\
\hline & & & & Water & 1.2 & $>100$ \\
\hline & Parkinsonia aculeata L. & BP01144 & Twigs & $\mathrm{DCM} / \mathrm{MeOH}(1: 1)$ & 0.3 & 9 \\
\hline & & & & Water & 0.8 & $>100$ \\
\hline & $\begin{array}{l}\text { Piliostigma thonningii } \\
\text { (Schumach.) Milne-Redh. }\end{array}$ & BР01294 & Leaves & DCM/MeOH (1:1) & 0.9 & 32 \\
\hline & & & & Water & 1.0 & $>100$ \\
\hline & & BP01294 & Fruit & $\mathrm{DCM} / \mathrm{MeOH}(1: 1)$ & 0.3 & 32.4 \\
\hline & & & & Water & 4.1 & 59 \\
\hline & & BP01294 & Twigs & $\mathrm{DCM} / \mathrm{MeOH}(1: 1)$ & 0.3 & 25.9 \\
\hline & & & & Water & 0.8 & $>100$ \\
\hline & $\begin{array}{l}\text { Pseudarthria hookeri Wight \& } \\
\text { Arn. var. hookeri }\end{array}$ & ВР01217 & Leaves & DCM/MeOH (1:1) & 1.3 & 100 \\
\hline & & & & Water & 4.8 & $>100$ \\
\hline & Pterocarpus angolensis DC. & EN00083 & Stems & DCM & 0.7 & 15 \\
\hline & & & & DCM/MeOH (1:1) & 1.1 & 60 \\
\hline & & & & $\mathrm{MeOH}$ & 0.9 & 71 \\
\hline & Pterocarpus angolensis DC. & EN00083 & Stems & Water & 1.8 & $>100$ \\
\hline & & EN00083 & Roots & $\mathrm{DCM}$ & 0.9 & 10.6 \\
\hline & & & & $\mathrm{DCM} / \mathrm{MeOH}(1: 1)$ & 0.9 & 25.5 \\
\hline & & & & $\mathrm{MeOH}$ & 1.5 & 33.5 \\
\hline & & & & Water & 2.0 & 80 \\
\hline & Senna didymobotrya (Fresen.) & BP01078 & Leaves & $\mathrm{DCM} / \mathrm{MeOH}(1: 1)$ & 1.7 & 40 \\
\hline & Irwin \& Barneby & & & & & \\
\hline & & & & Water & 3.8 & $>100$ \\
\hline & & BP01078 & Twigs & DCM/MeOH (1:1) & 0.7 & 9.5 \\
\hline & & & & Water & 1.6 & $>100$ \\
\hline & & BP01078 & Pods & $\mathrm{DCM} / \mathrm{MeOH}(1: 1)$ & 0.3 & 18 \\
\hline & & & & Water & 2.5 & $>100$ \\
\hline & Senna petersiana (Bolle) Lock & BP01290 & Leaves & $\mathrm{DCM} / \mathrm{MeOH}(1: 1)$ & 2.7 & $>100$ \\
\hline & & & & Water & 6.2 & $>100$ \\
\hline & & BP01290 & Twigs & $\mathrm{DCM} / \mathrm{MeOH}(1: 1)$ & 0.3 & 13 \\
\hline & & & & Water & 0.7 & $>100$ \\
\hline \multirow[t]{4}{*}{ Flacourtiaceae } & Flacourtia indica (Burm. f.) & EN0029 & Roots & DCM & 0.7 & 86.5 \\
\hline & Merr. & & & & & \\
\hline & & & & $\mathrm{DCM} / \mathrm{MeOH}(1: 1)$ & 1.3 & 78 \\
\hline & & & & Water & 0.9 & $>100$ \\
\hline \multirow[t]{4}{*}{ Gentianaceae } & Anthocleista grandiflora Gilg & NB00028 & Leaves & DCM & 1.4 & $>100$ \\
\hline & & & & DCM/MeOH (1:1) & 1.2 & $>100$ \\
\hline & & & & Water & 2.6 & 90 \\
\hline & $\begin{array}{l}\text { Pelargonium alchemilloides (L.) } \\
\text { 'Hér }\end{array}$ & BP01018 & Whole plant & DCM/MeOH $(1: 1)$ & 0.7 & 15 \\
\hline
\end{tabular}


Table 1 (Continued)

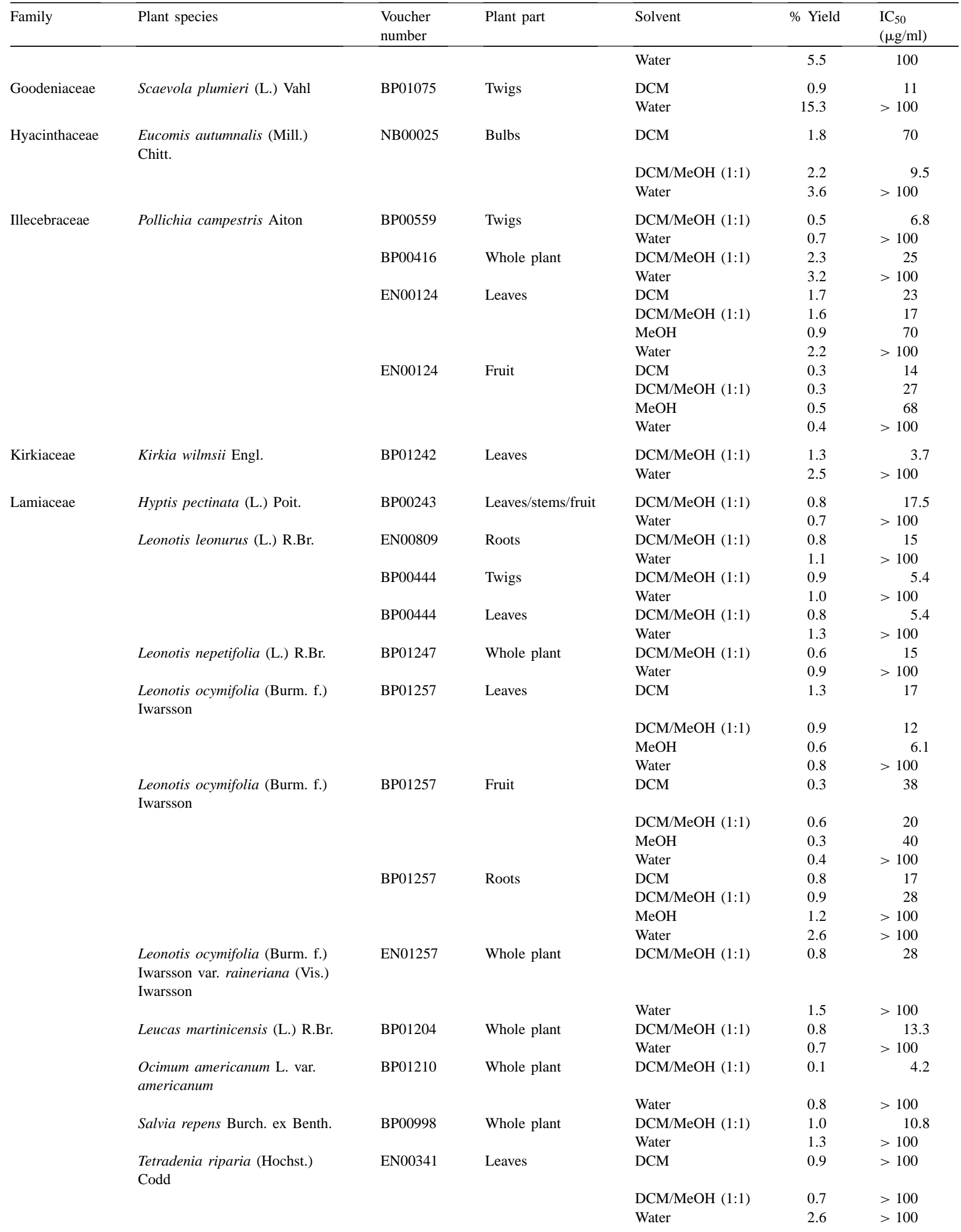


Table 1 (Continued)

\begin{tabular}{|c|c|c|c|c|c|c|}
\hline Family & Plant species & $\begin{array}{l}\text { Voucher } \\
\text { number }\end{array}$ & Plant part & Solvent & $\%$ Yield & $\begin{array}{l}\mathrm{IC}_{50} \\
(\mu \mathrm{g} / \mathrm{ml})\end{array}$ \\
\hline \multirow[t]{4}{*}{ Lecythidaceae } & $\begin{array}{l}\text { Barringtonia racemosa (L.) } \\
\text { Roxb. }\end{array}$ & BP00919 & Leaves & $\mathrm{DCM} / \mathrm{MeOH}(1: 1)$ & 1.2 & 18 \\
\hline & & & & Water & 4.0 & 70 \\
\hline & & ВР00919 & Twigs & DCM/MeOH (1:1) & 0.5 & 5.7 \\
\hline & & & & Water & 1.7 & $>100$ \\
\hline \multirow[t]{2}{*}{ Maesaceae } & Maesa lanceolata Forssk. & ВP01226 & Twigs & DCM/MeOH (1:1) & 0.7 & 5.9 \\
\hline & & & & Water & 0.2 & $>100$ \\
\hline \multirow[t]{8}{*}{ Meliaceace } & Ekebergia capensis Sparrm. & BP00442 & Fruit & DCM/MeOH (1:1) & 0.4 & 10 \\
\hline & & & & Water & 0.6 & $>100$ \\
\hline & & BP00442 & Twigs & DCM/MeOH (1:1) & 0.5 & 18 \\
\hline & Ekebergia capensis Sparrm. & ВР00979 & Twigs & Water & 0.8 & $>100$ \\
\hline & $\begin{array}{l}\text { Trichilia emetica Vahl } \\
\text { subsp. emetica }\end{array}$ & BP00958 & Leaves/twigs & DCM/MeOH (1:1) & 3.4 & 3.5 \\
\hline & & & & Water & 2.2 & $>100$ \\
\hline & Turraea floribunda Hochst. & BP01265 & Leaves & DCM/MeOH (1:1) & 0.5 & 8.8 \\
\hline & & & & Water & 2.8 & $>100$ \\
\hline \multirow[t]{4}{*}{ Myrtaceae } & $\begin{array}{l}\text { Syzigium cordatum Hochst. ex } \\
\text { Sond. var. cordatum }\end{array}$ & BP01268 & Twigs & DCM/MeOH (1:1) & 1.8 & 14.7 \\
\hline & & & & Water & 1.3 & 48.3 \\
\hline & & BP01268 & Leaves & DCM/MeOH (1:1) & 0.7 & 22.8 \\
\hline & & & & Water & 0.9 & 23.6 \\
\hline \multirow[t]{4}{*}{ Myrsinaceae } & $\begin{array}{l}\text { Rapanea melanophloeos (L.) } \\
\text { Mez }\end{array}$ & BP01031 & Leaves & DCM/MeOH (1:1) & 2.0 & 44 \\
\hline & & & & Water & 1.2 & $>100$ \\
\hline & & BP01031 & Twigs & DCM/MeOH (1:1) & 1.0 & 40 \\
\hline & & & & Water & 0.9 & $>100$ \\
\hline \multirow[t]{8}{*}{ Olacaceae } & Ximenia caffra Sond. var. caffra & EN00110 & Leaves & $\mathrm{DCM}$ & 0.7 & 43.5 \\
\hline & & & & DCM/MeOH (1:1) & 0.9 & 55 \\
\hline & & & & $\mathrm{MeOH}$ & 0.7 & 100 \\
\hline & & & & Water & 2.3 & $>100$ \\
\hline & & EN00110 & Roots & DCM & 0.5 & $>100$ \\
\hline & & & & DCM/MeOH (1:1) & 0.7 & $>100$ \\
\hline & & & & $\mathrm{MeOH}$ & 1.0 & $>100$ \\
\hline & & & & Water & 1.9 & $>100$ \\
\hline \multirow[t]{4}{*}{ Oleaceae } & $\begin{array}{l}\text { Olea europaea L. } \\
\text { subsp. africana (Mill.) P.S. } \\
\text { Green }\end{array}$ & BP01236 & Leaves & $\mathrm{DCM} / \mathrm{MeOH}(1: 1)$ & 0.7 & 12 \\
\hline & & & & Water & 0.7 & $>100$ \\
\hline & & BP01236 & Twigs & DCM/MeOH (1:1) & 1.2 & 13 \\
\hline & & & & Water & 1.1 & $>100$ \\
\hline \multirow[t]{8}{*}{ Pittosporaceae } & Pittosporum viridiflorum Sims & EN00049 & Whole plant & $\mathrm{DCM}$ & 1.5 & 3 \\
\hline & & & & DCM/MeOH (1:1) & 0.9 & 10 \\
\hline & & & & $\mathrm{MeOH}$ & 0.6 & 27.7 \\
\hline & Pittosporum viridiflorum Sims & EN00049 & Whole plant & Water & 1.4 & $>100$ \\
\hline & & EN00049 & Leaves/flowers & DCM & 0.8 & 28 \\
\hline & & & & DCM/MeOH (1:1) & 0.6 & 47 \\
\hline & & & & $\mathrm{MeOH}$ & 0.8 & 70.5 \\
\hline & & & & Water & 1.7 & $>100$ \\
\hline \multirow[t]{3}{*}{ Plantaginaceae } & Plantago major L. & EN00499 & Whole plant & DCM & 0.9 & 21.5 \\
\hline & & & & DCM/MeOH (1:1) & 1.2 & 45 \\
\hline & & & & Water & 1.9 & $>100$ \\
\hline \multirow[t]{8}{*}{ Plumbaginaceae } & Plumbago zeylanica L. & EN00208 & Roots & $\mathrm{DCM}$ & 0.8 & 43 \\
\hline & & & & DCM/MeOH (1:1) & 1.3 & 34 \\
\hline & & & & $\mathrm{MeOH}$ & 1.1 & 77.3 \\
\hline & & & & Water & 2.4 & $>100$ \\
\hline & & EN00208 & Leaves & DCM & 1.0 & 3 \\
\hline & & & & DCM/MeOH (1:1) & 0.7 & 4.8 \\
\hline & & & & $\mathrm{MeOH}$ & 0.9 & 5.5 \\
\hline & & & & Water & 1.9 & $>100$ \\
\hline
\end{tabular}


Table 1 (Continued)

\begin{tabular}{|c|c|c|c|c|c|c|}
\hline Family & Plant species & $\begin{array}{l}\text { Voucher } \\
\text { number }\end{array}$ & Plant part & Solvent & $\%$ Yield & $\begin{array}{l}\mathrm{IC}_{50} \\
(\mu \mathrm{g} / \mathrm{ml})\end{array}$ \\
\hline \multirow[t]{4}{*}{ Poaceae } & $\begin{array}{l}\text { Cymbopogon validus (Stapf) } \\
\text { Stapf ex Burtt Davy }\end{array}$ & ВР01227 & Whole plant & $\mathrm{MeOH} / \mathrm{DCM}(1: 1)$ & 0.9 & 5.8 \\
\hline & & & & Water & 0.8 & $>100$ \\
\hline & $\begin{array}{l}\text { Setaria megaphylla (Steud.) T. } \\
\text { Durand \& Schinz }\end{array}$ & BP01200 & Whole plant & $\mathrm{MeOH} / \mathrm{DCM}(1: 1)$ & 0.8 & 4.5 \\
\hline & & & & Water & 0.9 & $>100$ \\
\hline \multirow[t]{8}{*}{ Polygonaceae } & Rumex crispus $\mathrm{L}$. & EN00569 & Leaves & $\mathrm{DCM}$ & 1.2 & 36.8 \\
\hline & & & & DCM/MeOH (1:1) & 1.5 & 60 \\
\hline & & & & Water & 0.9 & $>100$ \\
\hline & & EN00569 & Roots & DCM & 0.9 & 14 \\
\hline & & & & $\mathrm{DCM} / \mathrm{MeOH}(1: 1)$ & 0.7 & 32 \\
\hline & & & & Water & 1.8 & $>100$ \\
\hline & Rumex sagittatus Thunb. & BP01201 & Whole plant & DCM/MeOH (1:1) & 0.5 & 18 \\
\hline & & & & Water & 0.1 & 100 \\
\hline \multirow[t]{9}{*}{ Ptaeroxylaceae } & $\begin{array}{l}\text { Ptaeroxylon obliquum (Thunb.) } \\
\text { Radlk. }\end{array}$ & EN00646 & Roots & DCM & 0.6 & 19 \\
\hline & & & & DCM/MeOH (1:1) & 0.9 & 17 \\
\hline & & & & Water & 1.3 & $>100$ \\
\hline & & EN00646 & Leaves & $\mathrm{DCM}$ & 0.8 & 19.5 \\
\hline & & & & DCM/MeOH (1:1) & 0.5 & 22.8 \\
\hline & & & & Water & 0.6 & $>100$ \\
\hline & & EN00646 & Stems & DCM & 0.7 & 11.5 \\
\hline & & & & DCM/MeOH (1:1) & 0.8 & 5.5 \\
\hline & & & & Water & 1.2 & $>100$ \\
\hline \multirow[t]{4}{*}{ Ranunculaceae } & Clematis brachiata Thunb. & ВP00192 & Leaves/stems/flowers & DCM/MeOH (1:1) & 0.8 & 20 \\
\hline & & & & Water & 3.0 & $>100$ \\
\hline & Ranunculus multifidus Forssk. & BP00962 & Whole plant & DCM/MeOH (1:1) & 0.2 & 2.3 \\
\hline & & & & Water & 2.7 & $>100$ \\
\hline \multirow[t]{4}{*}{ Rhamnaceae } & Ziziphus mucronata Willd. & BP00005 & Leaves & DCM & 0.8 & 12 \\
\hline & & & & $\mathrm{DCM} / \mathrm{MeOH}(1: 1)$ & 1.3 & $>100$ \\
\hline & & & & $\mathrm{MeOH}$ & 0.4 & $>100$ \\
\hline & & & & Water & 0.8 & $>100$ \\
\hline \multirow[t]{8}{*}{ Rhizophoraceae } & Bruguiera gymnorhiza (L.) Lam. & BP01275 & Twigs & DCM/MeOH (1:1) & 0.6 & 11.7 \\
\hline & & & & Water & 0.9 & $>100$ \\
\hline & & BP01275 & Leaves & DCM/MeOH (1:1) & 1.2 & 15.3 \\
\hline & & & & Water & 2.6 & $>100$ \\
\hline & Rhizophora mucronata Lam. & ВР01274 & Leaves & DCM/MeOH (1:1) & 0.7 & 24 \\
\hline & & & & Water & 4.5 & 25 \\
\hline & & BP01274 & Twigs & DCM/MeOH (1:1) & 0.6 & 5.6 \\
\hline & & & & Water & 2.2 & $>100$ \\
\hline \multirow[t]{9}{*}{ Rubiaceae } & Burchellia bubalina (L.f.) Sims & ВР00936 & Twigs & DCM/MeOH (1:1) & 0.4 & 18 \\
\hline & & & & Water & 1.3 & $>100$ \\
\hline & & BP00936 & Leaves & DCM/MeOH (1:1) & 0.8 & 50 \\
\hline & Cephalanthus natalensis Oliv. & BP01299 & Leaves & DCM/MeOH (1:1) & 0.2 & 24.3 \\
\hline & & & & Water & 3.6 & $>100$ \\
\hline & & BP01299 & Twigs & DCM/MeOH (1:1) & 0.1 & 16.5 \\
\hline & & & & Water & 1.4 & $>100$ \\
\hline & $\begin{array}{l}\text { Vangueria infausta Burch. } \\
\text { subsp. infausta }\end{array}$ & BP00004 & Fruit & $\mathrm{DCM} / \mathrm{MeOH}(1: 1)$ & 0.9 & 23 \\
\hline & & & & Water & 0.7 & $>100$ \\
\hline \multirow[t]{9}{*}{ Rutaceae } & Agathosma apiculata $\mathrm{G}$. Mey. & BP01123 & Whole plant & $\mathrm{DCM} / \mathrm{MeOH}(1: 1)$ & 1.1 & 5.2 \\
\hline & & & & Water & 1.9 & $>100$ \\
\hline & Agathosma puberula (Steud.) & EN00697 & Roots & $\mathrm{DCM}$ & 0.8 & 33 \\
\hline & Forc. & & & & & \\
\hline & & & & DCM/MeOH (1:1) & 0.7 & 19 \\
\hline & & & & Water & 1.4 & $>100$ \\
\hline & & EN00697 & Stems & $\mathrm{DCM}$ & 0.9 & 15 \\
\hline & & & & DCM/MeOH (1:1) & 0.8 & 15 \\
\hline & & & & Water & 1.6 & $>100$ \\
\hline
\end{tabular}


Table 1 (Continued)

\begin{tabular}{|c|c|c|c|c|c|c|}
\hline Family & Plant species & $\begin{array}{l}\text { Voucher } \\
\text { number }\end{array}$ & Plant part & Solvent & $\%$ Yield & $\begin{array}{l}\mathrm{IC}_{50} \\
(\mu \mathrm{g} / \mathrm{ml})\end{array}$ \\
\hline & $\begin{array}{l}\text { Clausena anisata (Willd.) Hook. } \\
\text { f. ex Benth var. anisata }\end{array}$ & BP01125 & Twigs & DCM/MeOH $(1: 1)$ & 0.6 & 18 \\
\hline & & & & Water & 1.0 & $>100$ \\
\hline & & BP01125 & Leaves & $\mathrm{DCM} / \mathrm{MeOH}(1: 1)$ & 1.0 & 55 \\
\hline & & & & Water & 2.9 & $>100$ \\
\hline & Diosma sp. & EN00735 & Roots & DCM & 0.4 & 10 \\
\hline & & & & DCM/MeOH (1:1) & 0.8 & 80 \\
\hline & & & & Water & 1.2 & $>100$ \\
\hline & $\begin{array}{l}\text { Macrostylis squarrosa Bartl. \& } \\
\text { H.L. Wendl. }\end{array}$ & EN00758 & Stems & $\mathrm{DCM} / \mathrm{MeOH}(1: 1)$ & 0.6 & 10 \\
\hline & & & & Water & 0.7 & $>100$ \\
\hline \multirow[t]{10}{*}{ Sapindaceae } & Cardiospermum halicacabum $\mathrm{L}$. & BP01286 & Whole plant & $\mathrm{DCM} / \mathrm{MeOH}(1: 1)$ & 1.5 & 20 \\
\hline & & & & Water & 2.8 & $>100$ \\
\hline & Dodonaea viscosa Jacq. & EN00791 & Leaves & DCM/MeOH (1:1) & 0.5 & 15.5 \\
\hline & & & & Water & 0.7 & $>100$ \\
\hline & Hippobromus pauciflorus (L.f.) & BP01229 & Leaves & $\mathrm{DCM} / \mathrm{MeOH}(1: 1)$ & 0.4 & 34 \\
\hline & Radlk. & & & & & \\
\hline & Hippobromus pauciflorus (L.f.) & BP01229 & Leaves & Water & 0.2 & 60 \\
\hline & Radlk. & & & & & \\
\hline & & BP01229 & Twigs & $\mathrm{DCM} / \mathrm{MeOH}(1: 1)$ & 0.6 & 5.9 \\
\hline & & & & Water & 0.9 & $>100$ \\
\hline \multirow[t]{4}{*}{ Sterculiaceae } & Hermannia depressa N.E.Br. & BP01081 & Whole plant & $\mathrm{DCM} / \mathrm{MeOH}(1: 1)$ & 0.4 & 6.9 \\
\hline & & & & Water & 4.1 & 70 \\
\hline & Waltheria indica $\mathrm{L}$. & BP01241 & Whole plant & $\mathrm{DCM} / \mathrm{MeOH}(1: 1)$ & 0.7 & $>100$ \\
\hline & & & & Water & 2.2 & $>100$ \\
\hline \multirow[t]{15}{*}{ Strychnaceae } & $\begin{array}{l}\text { Strychnos madagascariensis } \\
\text { Poir. }\end{array}$ & EN00286 & Stems & DCM & 0.8 & 70 \\
\hline & & & & DCM/MeOH (1:1) & 0.9 & 31.5 \\
\hline & & & & Water & 2.6 & 85 \\
\hline & & EN00286 & Leaves & $\mathrm{DCM}$ & 0.5 & 65 \\
\hline & & & & $\mathrm{DCM} / \mathrm{MeOH}(1: 1)$ & 0.8 & 40 \\
\hline & & & & Water & 1.3 & 74 \\
\hline & & EN00286 & Roots & DCM & 0.7 & 56 \\
\hline & & & & DCM/MeOH (1:1) & 0.9 & $>100$ \\
\hline & & & & Water & 1.2 & $>100$ \\
\hline & Strychnos potatorum L.f. & EN00219 & Leaves & DCM & 0.9 & 60 \\
\hline & & & & $\mathrm{DCM} / \mathrm{MeOH}(1: 1)$ & 1.3 & $>100$ \\
\hline & Strychnos pungens Soler. & EN00186 & Leaves & DCM & 0.7 & 12.6 \\
\hline & & & & DCM/MeOH (1:1) & 0.8 & 80.4 \\
\hline & & & & $\mathrm{MeOH}$ & 0.8 & 80 \\
\hline & & & & Water & 2.3 & 39 \\
\hline \multirow[t]{10}{*}{ Thymelaeaceae } & Gnidia cuneata Meisn. & EN00716 & Leaves & DCM & 0.7 & 31.1 \\
\hline & & & & DCM/MeOH (1:1) & 0.9 & 51 \\
\hline & & & & Water & 1.4 & 45.5 \\
\hline & & EN00716 & Stems & $\mathrm{DCM}$ & 0.6 & 15.9 \\
\hline & & & & $\mathrm{DCM} / \mathrm{MeOH}(1: 1)$ & 0.8 & 40.5 \\
\hline & & & & Water & 1.5 & $>100$ \\
\hline & $\begin{array}{l}\text { Gnidia kraussiana Meisn. var. } \\
\text { kraussiana }\end{array}$ & BP01008 & Tuber & $\mathrm{DCM} / \mathrm{MeOH}(1: 1)$ & 0.7 & 16 \\
\hline & & & & Water & 4.4 & $>100$ \\
\hline & & ВР01008 & Leaves/twigs & $\mathrm{DCM} / \mathrm{MeOH}(1: 1)$ & 0.4 & 10.8 \\
\hline & & & & Water & 1.3 & $>100$ \\
\hline \multirow[t]{2}{*}{ Tiliaceae } & $\begin{array}{l}\text { Triumfetta welwitschii Mast. var. } \\
\text { hirsuta (Sprague \& Hutch.) Wild }\end{array}$ & ВР01238 & Leaves & $\mathrm{DCM} / \mathrm{MeOH}(1: 1)$ & 1.0 & 3.6 \\
\hline & & & & Water & 1.7 & $>100$ \\
\hline \multirow[t]{4}{*}{ Verbenaceae } & $\begin{array}{l}\text { Clerodendrum glabrum E. Mey. } \\
\text { var. glabrum }\end{array}$ & BP01207 & Twigs & $\mathrm{MeOH} / \mathrm{DCM}(1: 1)$ & 0.1 & 19 \\
\hline & & & & Water & 3.5 & $>100$ \\
\hline & Lantana camara L. & ВР01216 & Leaves/twigs & DCM/MeOH (1:1) & 1.3 & 11 \\
\hline & & & & Water & 4.8 & $>100$ \\
\hline
\end{tabular}


Table 1 (Continued)

\begin{tabular}{|c|c|c|c|c|c|c|}
\hline Family & Plant species & $\begin{array}{l}\text { Voucher } \\
\text { number }\end{array}$ & Plant part & Solvent & $\%$ Yield & $\begin{array}{l}\mathrm{IC}_{50} \\
(\mu \mathrm{g} / \mathrm{ml})\end{array}$ \\
\hline & $\begin{array}{l}\text { Lippia javanica (Burm. f.) } \\
\text { Spreng. }\end{array}$ & ВР00200 & Roots & DCM & 0.8 & 3.8 \\
\hline & & & & DCM/MeOH (1:1) & 1.3 & 27 \\
\hline & & & & $\mathrm{MeOH}$ & 0.7 & 24 \\
\hline & & & & Water & 0.6 & $>100$ \\
\hline & & ВР00200 & Stems & DCM & 0.6 & 4.5 \\
\hline & & & & DCM/MeOH (1:1) & 1.7 & 21.8 \\
\hline & & & & $\mathrm{MeOH}$ & 0.8 & 29.8 \\
\hline & & & & Water & 1.0 & $>100$ \\
\hline
\end{tabular}

(Celastraceae) (Brenniesen and Geisshusler, 1985; Carlini, 2003), Conyza albida Spreng. (Asteraceae) (Pacciaroni et al., 2000; Stamatis et al., 2003), Lippia javanica (Burm. f.) Spreng. (Verbenaceae) (Neidlein and Staehle, 1974; Van Wyk et al., 1997) and Ocimum americanum L. var. americanum (Lamiaceae) (Vieira et al., 2003), there are no reports on their antiplasmodial activity. Several representatives of genera investigated here (viz. Croton (Euphorbiaceae) (Prozesky et al., 2001), Acacia (Fabaceae) (El Tahir et al., 1999b), Hypericum (Clusiaceae) (Decosterd et al., 1991; Gu et al., 1988), Triumfetta (Tiliaceae) (Muñoz et al., 2000) and Vernonia (Asteraceae) (Abosi and Raseroka, 2003; Kraft et al., 2003; Oketch-Rabah et al., 1998; Alves et al., 1997)) have previously been shown to have antiplasmodial

Table 2

Native or naturalised South African plants with high antiplasmodial activity, and comments on prior reports

\begin{tabular}{|c|c|c|}
\hline & $\mathrm{IC}_{50}(\mu \mathrm{g} / \mathrm{ml})$ & Comments \\
\hline \multicolumn{3}{|l|}{ Taxon } \\
\hline Acacia tortilis & 4.8 & Acacia nilotica (L.) ex Delile has shown in vitro activity (El Tahir et al., 1999b) \\
\hline Aloe marlothii & 3.5 & $\begin{array}{l}\text { In vitro activity previously reported (Van Zyl and Viljoen, 2002), although 16-fold lower than } \\
\text { observed in this study }\end{array}$ \\
\hline Artemisia afra & 5.0 & $\begin{array}{l}\text { In vitro activity previously reported; active constituents do not appear to be artemisinin-type } \\
\text { compounds (Kraft et al., 2003) }\end{array}$ \\
\hline Bidens pilosa $a^{\mathrm{a}}$ & 5.0 & $\begin{array}{l}\text { Watt and Breyer-Brandwijk (1962) reported negative antimalarial findings, but more recent tests } \\
\text { yielded positive in vitro results (Brandão et al., 1997; Krettli et al., 2001) }\end{array}$ \\
\hline Catha edulis & 0.6 & No previous reports of antiplasmodial activity \\
\hline Conyza albida ${ }^{\mathrm{a}}$ & 2.0 & No previous reports of antiplasmodial activity \\
\hline Croton gratissimus & 3.5 & Croton pseudopulchellus Pax has shown in vitro activity (Prozesky et al., 2001) \\
\hline Croton menyhartii & 1.7 & Croton pseudopulchellus Pax has shown in vitro activity (Prozesky et al., 2001) \\
\hline Euclea undulata & 4.6 & No previous reports of antiplasmodial activity \\
\hline Hypericum aethiopicum & 1.4 & $\begin{array}{l}\text { Hypericum japonicum Thunb. (Gu et al., 1988) and Hypericum calcyinum L. (Decosterd et al., } \\
\text { 1991) have shown in vivo and in vitro activity }\end{array}$ \\
\hline Kirkia wilmsii & 3.7 & No previous reports of antiplasmodial activity \\
\hline Lippia javanica & 3.8 & $\begin{array}{l}\text { Reported to be a mosquito repellent (Govere et al., 2000), but no previous reports of } \\
\text { antiplasmodial activity }\end{array}$ \\
\hline Oсітит атеricanum ${ }^{\mathrm{a}}$ & 4.2 & $\begin{array}{l}\text { Used as a mosquito repellent (Seyoum et al., 2002), but no previous reports of antiplasmodial } \\
\text { activity }\end{array}$ \\
\hline Pittosporum viridiflorum & 3.0 & No previous reports of antiplasmodial activity \\
\hline Plumbago zeylanica & 3.0 & In vitro activity previously reported (Simonsen et al., 2001) \\
\hline Ranunculus multifidus & 2.3 & No previous reports of antiplasmodial activity \\
\hline Schefflera umbellifera & 3.7 & In vitro activity previously reported (Tetyana et al., 2002) \\
\hline \multicolumn{3}{|l|}{ Species } \\
\hline Setaria megaphylla & 4.5 & No previous reports of antiplasmodial activity \\
\hline Trichilia emetica & 3.5 & $\begin{array}{l}\text { Trichilia emetica and other members of genus have shown in vitro activity (MacKinnon et al., } \\
\text { 1997; El Tahir et al., 1999b; Prozesky et al., 2001) }\end{array}$ \\
\hline Triumfetta welwitschii & 3.6 & Triumfetta semitrilobata Jacq. has shown in vitro and in vivo activity (Muñoz et al., 2000) \\
\hline Vernonia colarata & 4.7 & $\begin{array}{l}\text { Several Vernonia species have shown activity in vitro and in vivo (Alves et al., } \\
\text { 1997; Oketch-Rabah et al., 1998; Abosi and Raseroka, 2003; Kraft et al., 2003) }\end{array}$ \\
\hline Vernonia myriantha & 3.0 & $\begin{array}{l}\text { Several Vernonia species have shown activity in vitro and in vivo (Alves et al., } \\
\text { 1997; Oketch-Rabah et al., 1998; Abosi and Raseroka, 2003; Kraft et al., 2003) }\end{array}$ \\
\hline Vernonia oligocephala & 3.5 & $\begin{array}{l}\text { Several Vernonia species have shown activity in vitro and in vivo (Alves et al., 1997; } \\
\text { Oketch-Rabah et al., 1998; Abosi and Raseroka, 2003; Kraft et al., 2003) }\end{array}$ \\
\hline
\end{tabular}

\footnotetext{
${ }^{a}$ Naturalised in South Africa.
} 
activity. In addition to identifying further species within these genera that display activity, our findings are substantiated by earlier reported antiplasmodial activity elsewhere in the genera. Similarly, the results for Plumbago zeylanica L. (Plumbaginaceae) (Simonsen et al., 2001), Bidens pilosa L. (Asteraceae) (Brandão et al., 1997; Krettli et al., 2001), Trichilia emetica Vahl (Meliaceae) (El Tahir et al., 1999a; Prozesky et al., 2001), Schefflera umbellifera (Sond.) Baill. (Araliaceae) (Tetyana et al., 2002) and Artemisia afra Jacq. ex Willd. (Asteraceae) (Kraft et al., 2003) agree with previous reports on their antiplasmodial activity. The activity of Aloe marlothii A. Berger (Asphodelaceae) has been reported on before $\left(\mathrm{IC}_{50}>50 \mu \mathrm{g} / \mathrm{ml}\right.$ ), although it was considerably lower than that observed in this study (Van Zyl and Viljoen, 2002). A number of the genera investigated, namely, Euclea (Ebenaceae), Kirkia (Kirkiaceae), Pittosporum (Pittosporaceae), Ranunculus (Ranunculaceae) and Setaria (Poaceae) were shown for the first time to display high antiplasmodial activity. To the best of our knowledge, this is the first report of the antiplasmodial activity in the families Ebenaceae, Kirkiaceae and Pittosporaceae.

It is interesting to note that the majority of the aqueous extracts, which would be the preferred method of preparing the plants when used in traditional medicines, did not show any activity. Moreover, they would contain very little if any of the lipophilic compounds extracted with the organic solvents (DCM and $\mathrm{MeOH}$ ), which showed the greatest activity. A possible explanation for the poor hit rate of aqueous extracts is that they were not prepared according to the traditional methods, which often involves boiling for several hours. Furthermore, in the traditional context it is not uncommon for several plant taxa to be administered as mixtures. In this case, synergism could exist between the various phytochemicals or different constituents could help extract and keep active lipophilic compounds in an aqueous solution. In the course of this study the above-mentioned factors were not taken into consideration and only the in vitro antiplasmodial activity of individual plant extracts was determined.

A number of the plants selected did not display in vitro antiplasmodial activity, despite strong associations with malaria and its treatment. A possible explanation could be that the plants act as antipyretics or immune stimulants to relieve the symptoms of the disease, rather than having direct antiparasitic activity (Phillipson et al., 1993). Alternatively, precursors of the active components may be present in the extracts but have to be modified, usually in vivo, before activity is exhibited. Furthermore, factors such as chemotypes, environmental parameters, harvesting and storage conditions could collectively influence the plant secondary metabolites prior to and following harvesting, which in turn would be reflected in the bioactivity. Despite these possible sources of variability, a number of the plant species that were found to be active in this study have previously been shown to possess in vitro, and in some cases in vivo, antiplasmodial activity. In the course of this study, several taxa at the genus and family levels were shown for the first time to display in vitro antiplasmodial activity and warrant further investigation as potential sources of antiplasmodial agents. Additional in vitro and in vivo work aimed at understanding the mechanisms of action of the active plant species and isolating and characterising the bioactive constituents is underway in our laboratories and will be reported on in due course.

\section{Conclusions}

Not only has this study highlighted promising taxa for further antimalarial investigation, it has provided compelling evidence for the rational exploration of indigenous and naturalised South African medicinal plants as a source of antiplasmodial agents. Considering that most regional plant taxa have not been investigated chemically or pharmacologically, they remain a potential source of leads for drug development. This is particularly important for diseases lacking effective chemotherapeutic agents, such as malaria.

\section{Acknowledgements}

We gratefully acknowledge the Department of Science and Technology of South Africa for the Innovation Fund Grant (Project 31313). We wish to acknowledge the traditional knowledge holders of South Africa for their contribution to the indigenous knowledge systems of South Africa, and the many genetic resource holders who allowed access to their plant materials. In particular, N. Hahn and E. Schmidt are thanked for facilitating collections on their properties. In this regard, we also thank South Africa's provincial conservation authorities for permitting plant collections. We wish to thank the research and field teams of participating institutions. Miss S. Luwaca is thanked for administrative support.

\section{References}

Abosi, A.O., Raseroka, B.H., 2003. In vivo antimalarial activity of Vernonia amygdalina. British Medical Journal of Biomedical Science 60 , 89-91.

Alves, T.M., Nagem, T.J., de Carvalho, L.H., Krettli, A.U., Zani, C.L., 1997. Antiplasmodial triterpenes from Vernonia brasiliana. Planta Medica 63, 554-555.

Arnold, T.H., Prentice, C.A., Hawker, L.C., Snyman, E.E., Tomalin, M., Crouch, N.R., Pottas-Bircher, C., 2002. Medicinal and Magical Plants of Southern Africa: An Annotated Checklist. Strelitzia 13. National Botanical Institute, Pretoria, pp. 1-2.

Brandão, M.G.L., Krettli, A.U., Soares, L.S.R., Nery, C.G.C., Marinuzzi, H.C., 1997. Antimalarial activity of extracts and fractions from Bidens pilosa and Bidens species (Asteraceae) correlated with the presence of acetylene and flavonoid compounds. Journal of Ethnopharmacology 57, 131-138.

Brenniesen, R., Geisshusler, S., 1985. Psychotropic drugs III: analytical and chemical aspects of Catha edulis Forsk. Pharmacetica Acta Halvetiae $60,290-301$. 
Carlini, E.A., 2003. Plants and the central nervous system. Pharmacology, Biochemistry and Behavior 75, 501-512.

Clark, T.E., Appleton, C.C., Drewes, S.E., 1997. A semi-quantitative approach to the selection of appropriate candidate molluscicides-a South African application. Journal of Ethnopharmacology 56, 1-13.

Clarkson, C., Campbell, W.E., Smith, P., 2003. In vitro antiplasmodial activity of abietane and totarane diterpenes isolated from Harpagophytum procumbens (Devil's Claw). Planta Medica 8, 720-724.

Decosterd, L.A., Hoffmann, E., Kyburz, R., Bray, D., Hostettmann, K., 1991. A new phloroglucinol derivative from Hypericum calcyinum with antifungal and in vitro antimalarial activity. Planta Medica 57, 548-551.

El Tahir, A., Satti, G.M., Khalid, S.A., 1999a. Antiplasmodial activity of selected Sudanese medicinal plants with emphasis on Maytenus senegalensis (Lam.) Exell. Journal of Ethnopharmacology 64, 227-233.

El Tahir, A., Satti, G.M., Khalid, S.A., 1999b. Antiplasmodial activity of selected Sudanese medicinal plants with emphasis on Acacia nilotica. Phytotherapy Research 13, 474-478.

Govere, J., Durrheim, D.N., Du Toit, N., Hunt, R.H., Coetzee, M., 2000. Local plants as repellents against Anopheles arabiensis, in Mpumalanga Province, South Africa. Central African Journal of Medicine 46, 213216.

Gu, G., Feng, S., Xiaoyan, W., 1988. Antimalarial constituents of Hypericum japonicum Thunb. Isolation and structure of japonicins A, B, C and D. Huaxue Xuebao 46, 246-251.

Kraft, C., Jenett-Siems, K., Siems, K., Jakupovic, J., Mavi, S., Bienzle, U., Eich, E., 2003. In vitro antiplasmodial evaluation of medicinal plants from Zimbabwe. Phytotherapy Research 17, 123-128.

Krettli, A.U., Andrade-Neto, V.F., Brandão, M.G.L., Ferrari, W.M.S., 2001. The search for new antimalarial drugs from plants used to treat fever and malaria or plants randomly selected: a review. Memorias do Instituto Oswaldo Cruz 96, 1033-1042.

MacKinnon, S., Durst, T., Arnason, J.T., Angerhofer, C., Pezzuto, J., Sanchez-Vindas, P.E., Poveda, L.J., Gbeassor, M., 1997. Antimalarial activity of tropical Meliaceae extracts and Gedunin derivatives. Journal of Natural Products 60, 336-341.

Makler, M.T., Ries, J.M., Williams, J.A., Bancroft, J.E., Piper, R.C., Gibbins, B.L., et al., 1995. Parasite lactate dehydrogenase as an assay for Plasmodium falciparum drug sensitivity. American Journal of Tropical Medicine and Hygiene 48, 739-741.

Muñoz, V., Sauvain, M., Bourdy, G., Callapa, J., Rojas, I., Vargas, L., Tae, A., Deharo, E., 2000. The search for natural bioactive compounds through a multidisciplinary approach in Bolivia. Part II. Antimalarial activity of some plants used by Mosetene Indians. Journal of Ethnopharmacology 69, 139-155.

Neidlein, R., Staehle, R., 1974. Constituents of Lippia javanica. III. Deutsche Apotheker Zeitung 114, 1588-1592.

Oketch-Rabah, H.A., Christensen, S.B., Frydenvang, K., Dossaji, S.F., Theander, T.G., Cornett, C., Watkins, W.M., Kharazmi, A., Lemmich,
E., 1998. Antiprotozoal properties of 16,17-dihydrobrachycalyxolide from Vernonia brachycalyx. Planta Medica 64, 559562.

Pacciaroni, A.V., Mongelli, E., Ariza Espinar, L., Romano, A., Ciccia, G., Silva, G.L., 2000. Bioactive constituents of Conyza albida. Planta Medica 66, 720-723.

Phillipson, J.D., Wright, C.W., Kirby, G.C., Warhurst, D.C., 1993. Tropical plants as sources of antiprotozoal agents. Recent Advances in Phytochemistry 27, 1-40.

Prozesky, E.A., Meyer, J.J.M., Louw, A.I., 2001. In vitro antiplasmodial activity and cytotoxicity of ethnobotanically selected South African plants. Journal of Ethnopharmacology 76, 239-245.

Ridley, R.G., 2002. Medical need, scientific opportunity and the drive for antimalarial drugs. Nature 415, 686-693.

Seyoum, A., Palsson, K., Kung'a, S., Kabiru, E.W., Lwande, W., Killeen, G.F., Hassanali, A., Knols, B.G.J., 2002. Traditional use of mosquito-repellent plants in western Kenya and their evaluation in semi-field experimental huts against Anopheles gambiae: ethnobotanical studies and application by thermal expulsion and direct burning. Transactions of the Royal Society of Medicine and Hygiene 96, 225231.

Simonsen, H.T., Nordskjold, J.B., Smitt, U.W., Nyman, U., Palpu, P., Joshi, P., Varughese, G., 2001. In vitro screening of Indian medicinal plants for antiplasmodial activity. Journal of Ethnopharmacology 74, 195-204.

Stamatis, G., Kyriazopoulos, P., Golegou, S., Basayiannis, A., Skaltsas, S., Skaltsa, H., 2003. In vitro anti-Helicobacter pylori activity of Greek herbal medicines. Journal of Ethnopharmacology 88, 175-179.

Tetyana, P., Prozesky, E.A., Jäger, A.K., Meyer, J.J.M., van Staden, J., 2002. Some medicinal properties of Cussonia and Schefflera species used in traditional medicine. South African Journal of Botany 68, 5154.

Trager, W., Jensen, J.B., 1976. Human malaria parasites in continuous culture. Science 193, 673-675.

Van Wyk, B.E., 2002. A review of ethnobotanical research in southern Africa. South African Journal of Botany 68, 1-13.

Van Wyk, B.E., van Oudtshoorn, B., Gericke, N., 1997. Medicinal Plants of South Africa. Briza Publications, Pretoria, South Africa.

Van Zyl, R.L., Viljoen, A.M., 2002. In vitro activity of Aloe extracts against Plasmodium falciparum. South African Journal of Botany 68, 106-110.

Vieira, R.F., Grayer, R.J., Paton, A.J., 2003. Chemical profiling of Ocimum americanum using external flavonoids. Phytochemistry 63, 555567.

Watt, J.M., Breyer-Brandwijk, M.G., 1962. The Medicinal and Poisonous Plants of Southern and Eastern Africa. E. \& S. Livingstone Ltd., Edinburgh, UK.

WHO, 2003. World Health Organisation Fact Sheet No. 94. WHO information. http://www.int/inf fs/en/fact094.html. 